\title{
The Effect of Water Accessible Paramagnetic Ions on Subcellular Structures Formed in Developing Wheat Photosynthetic Membranes as Observed by NMR and by Sorption Isotherm
}

\author{
H. HARAŃCZYK ${ }^{a, *}$, A. LeJA ${ }^{a}$ AND K. StrzaŁKA ${ }^{b}$ \\ ${ }^{a}$ Institute of Physics, Jagiellonian University \\ Reymonta 4, 30-059 Kraków, Poland \\ ${ }^{b}$ Faculty of Biotechnology, Jagiellonian University \\ Gronostajowa 7, 30-387 Kraków, Poland
}

The rehydration from the gaseous phase of the developing native or EDTA-washed from unbound and loosely bound paramagnetic ions wheat thylakoid membrane lyophilizate was investigated using hydration kinetics, sorption isotherm, and high power proton relaxometry. Hydration time courses are single exponential for all target humidities. The sorption isotherm is well fitted by the Dent model, with the mass of water saturating primary binding sites equal to $\Delta M / m_{0}=0.024$ and 0.017 for native and EDTA-washed membranes, respectively. Proton free induction decays distinguish: (i) a Gaussian component, $S_{0}$, coming from protons of solid matrix of lyophilizate; (ii) a Gaussian component, $S_{1}$, from water bound to the primary water binding sites in proximity of water accessible paramagnetic ions; (iii) an exponentially decaying contribution, $L_{1}$, from water tightly bound to lyophilizate surface; and (iv) exponentially decaying loosely bound water pool, $L_{2}$. Sorption isotherm fitted to NMR data shows a significant contribution of water "sealed" in membrane structures $\left(\Delta M_{s} / m_{0}=0.052\right.$ for native and 0.061 for EDTA-washed developing membranes, respectively).

PACS numbers: 82.56.Na, 87.16.Dg

\section{Introduction}

Plants differ in their susceptibility to drought stress, causing inhibition of the photosynthetic electron transport and perturbations of membrane structure [1]. Apart from number of species which are tolerant to a mild water deficit, there also

*corresponding author; e-mail: hubert.haranczyk@uj.edu.pl 
exist organisms resistant to deep desiccation (and frost), e.g. resurrection plants $[2,3]$ and lichens [4-8].

A good model for studying a recovery from the state of desiccation are freeze-dried photosynthetic membranes. It is not yet clear whether photosynthetic membrane lyophilizates retain lamellar phase, however, after rehydration they return to lamellar phase with the significant contribution of the small membrane fragments from the disrupted lamellae $[9,10]$.

A fundamental for understanding the molecular mechanism of recovery of metabolic activity of cell structures during rehydration is knowledge about number and distribution of water binding sites, sequence and kinetics of their saturation, as well as formation of tightly and loosely bound water pools at different steps of rehydration process. Moreover, in this paper we search for water "sealed" in membrane structures, and for the structural effect of paramagnetic ions bound to the rehydrated lyophilized photosynthetic membrane.

Although the dominating effect on proton relaxation in thylakoid membranes was assumed to come from the non-functional surface bound paramagnetic manganese ions [11-13], we consequently write just on paramagnetic ions. This is because the effect of other ion groups cannot be excluded as EDTA is a strong chelator and is not selective for paramagnetic ions.

\section{Materials and methods}

E class chloroplasts (photosynthetic lamellae purified from stroma proteins) were isolated from wheat (Triticum aestivum L.) var. Jara seedlings grown for six days in darkness and subsequently illuminated for $24 \mathrm{~h}$ with white light. The isolation procedure was as described previously [14], however, a $0.067 \mathrm{M}$ phosphate buffer at $\mathrm{pH} 7.3$ containing $0.5 \mathrm{M}$ sucrose was used instead of Tris-HCl buffer. Various unbound and loosely bound ions were removed by $1 \mathrm{mM}$ EDTA-washing for $18 \mathrm{~h}$ at $+4^{\circ} \mathrm{C}$. The obtained preparations of photosynthetic membranes were freeze-dried and stored desiccated at $+4^{\circ} \mathrm{C}$ in darkness. Prior to the hydration courses the lyophilizate was incubated for $48 \mathrm{~h}$ over silica gel, at the relative humidity $p / p_{0}=0 \%$.

The hydration time-courses were performed over the surface of $\mathrm{H}_{3} \mathrm{PO}_{4}$ $\left(p / p_{0}=9 \%\right)$, over the surfaces of supersaturated solutions of $\mathrm{CaCl}_{2}\left(p / p_{0}=32 \%\right)$, $\mathrm{Na}_{2} \mathrm{Cr}_{2} \mathrm{O}_{7}\left(p / p_{0}=52 \%\right), \mathrm{Na}_{2} \mathrm{~S}_{2} \mathrm{O}_{3}(76 \%), \mathrm{K}_{2} \mathrm{CrO}_{3}(88 \%), \mathrm{Na}_{2} \mathrm{SO}_{4}(93 \%)$, and over the water surface $\left(p / p_{0}=100 \%\right)$ at room temperature. As a measure of hydration level, the relative mass increase expressed in units of dry mass, $\Delta m / m_{0}$, was taken.

After completing the hydration courses, the dry mass of the photosynthetic membrane was determined after heating at $70^{\circ} \mathrm{C}$ for 68 hours. Higher temperatures were not used as they may decompose some organic constituents of the membrane [15].

Proton free induction decays (FIDs) were measured on high power relaxometer WNS-65 (Waterloo NMR Spectrometers, St. Agatha, Ontario, Canada) 
working at the resonance frequency $30 \mathrm{MHz}$. Transmitter power was $400 \mathrm{~W}$; pulse lengths $\pi / 2$ in the range between $1.25 \mu \mathrm{s}$ and $1.50 \mu \mathrm{s}$. Data were acquired using Compuscope 2000 card of an IBM 80486 clone, and averaged over 1000 accumulations. Repetition time was $2 \mathrm{~s}$.

The measurements were performed at room temperature $\left(t=22^{\circ} \mathrm{C}\right)$.

The recorded proton FIDs were decomposed using one-dimensional procedure of the program CracSpin (Jagiellonian University, Cracow, Poland) designed for time domain two-dimensional NMR data analysis [16].

\section{Results}

The hydration kinetics of lyophilized wheat photosynthetic membranes performed for the full range of target humidities (between 9\% and 100\%) (Fig. 1) was fitted well by the single exponential function

$$
\Delta m(t) / m_{0}=B^{\mathrm{h}}+A^{\mathrm{h}}\left[1-\exp \left(-t / t^{\mathrm{h}}\right)\right]
$$

where $t^{\mathrm{h}}$ is the hydration time, $B^{\mathrm{h}}$ is the saturation hydration at $p / p_{0}=0 \%$, and $A^{\mathrm{h}}$ is the hydration surplus at elevated air-humidity.
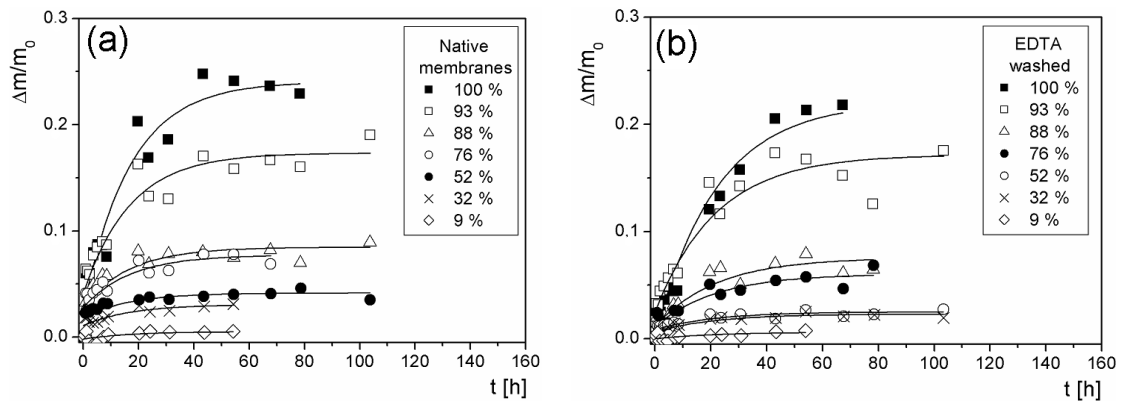

Fig. 1. The rehydration of wheat photosynthetic membrane lyophilizate from gaseous phase at different values of relative humidity, $p / p_{0}$, recorded at room temperature as relative mass increase, expressed in units of dry mass, $\Delta m / m_{0}$. (a) Native membranes, (b) EDTA-washed membranes.

The values averaged over the all target humidities, $B^{\mathrm{h}}$, were $0.022 \pm 0.005$ and $0.009 \pm 0.003$ for native and EDTA-washed membranes, respectively. There are noticeably lower values than those for the Antarctic lichens [17], suggesting the decreased density of primary water binding sites compared to lichen thallus surfaces. The hydration times equal $t^{\mathrm{h}}=(17.0 \pm 3.2) \mathrm{h}$ and $(22.0 \pm 2.8) \mathrm{h}$ for native and EDTA-washed membranes, respectively, which are the values similar to those for foliose thalli [17].

The saturation hydration level, $C^{\mathrm{h}}=A^{\mathrm{h}}+B^{\mathrm{h}}$, obtained from hydration kinetics was taken for adsorption isotherm calculation. For the wheat photosynthetic membrane lyophilizate rehydration the adsorption isotherm (Fig. 2) is sigmoidal in form [18] and is well described by the Dent model [19, 17]: 

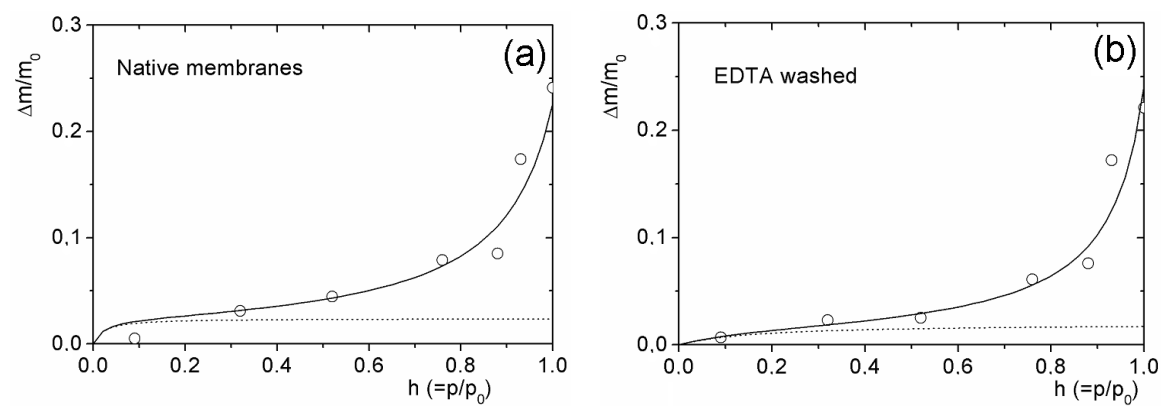

Fig. 2. The sorption isotherms recorded at room temperature for the lyophilizate of wheat photosynthetic membranes. Solid line shows total sorption, dotted line - the population of monolayer sorption predicted by the Dent model. $h$ is relative humidity expressed in absolute units. (a) Native membranes, (b) EDTA-washed membranes.

$$
C^{\mathrm{h}}(h)=\frac{\Delta M}{m_{0}} \frac{b_{1} h}{(1-b h)\left(1+b_{1} h-b h\right)},
$$

where $h=p / p_{0}$ is expressed as a fraction, and $\Delta M / m_{0}$ is the mass of water saturating primary binding sites. For native membranes $\Delta M / m_{0}=0.024$, whereas for EDTA-washed ones $\Delta M / m_{0}=0.017$, showing the elevated hydrophobicity of the surface compared to lichen thallus [17]. Another measure of surface hydrophobicity is parameter $1 / b_{1}$, which is a fraction of unbound primary binding sites at $h=1$. For native membranes $1 / b_{1}=2.2 \%$ and for EDTA-washed $1 / b_{1}=14.6 \%$, which means that removal of accidentally bound paramagnetic ions still decreases membrane affinity to water. Parameter $b$ is a coverage of the $n$-th water layer expressed in units of the coverage of $(n-1)$-th layer. For native membranes $b=0.896$, and for EDTA-washed $b=0.929$. Thus, binding to subsequent water layers does not differ much from BET model, where $b=1$ by definition [20]. The Dent model distinguishes two types of water binding sites: (i) "primary" binding sites - directly to the adsorbent surface, and (ii) "secondary", usually weaker, water binding sites, i.e. to primary bound water molecules, or to the next water layers. Although Dent introduced his model under the assumption that adsorbate molecules arrange in layers, this model still works if there is no layering of water bound to secondary binding sites.

Proton FID for developing wheat photosynthetic membranes is well described by the superposition of two Gaussian components and two exponents:

$$
\begin{aligned}
& \mathrm{FID}(t)=S_{0} \exp \left[-\left(\frac{t}{T_{S, 0}^{*}}\right)^{2}\right]+S_{1} \exp \left[-\left(\frac{t}{T_{S, 1}^{*}}\right)^{2}\right] \\
& \quad+L_{1} \exp \left(-\frac{t}{T_{L, 1}^{*}}\right)+L_{2} \exp \left(-\frac{t}{T_{L, 2}^{*}}\right)
\end{aligned}
$$

where $S_{0}$ and $S_{1}$ are the amplitudes of the Gaussian components, $T_{S, 0}^{*}$ and $T_{S, 1}^{*}$ 
are the 1/e-decay times for both Gaussian decays; $L_{1}$ and $L_{2}$ are the amplitudes of the exponential components, $T_{L, 1}^{*}$ and $T_{L, 2}^{*}$ are the relaxation times for both exponential components. For EDTA-washed membranes no $S_{1}$ component is detected. The 1/e-value allows a direct comparison between the characteristic time constant describing the solid and the ones describing the liquid signal components [21, 4, 22].

Figure 3 shows the hydration dependence of the proton relaxation times for the components of FID signal of rehydrated lyophilizates of wheat thylakoids. The time constant for the $S_{0}$ component does not depend on hydration level of the sample with the value $T_{S, 0}^{*} \approx 20 \mu \mathrm{s}$, which is close to the value for the solid matrices of bark and bast [23], lichen thallus [21, 4, 22], dentine and dental enamel [24]. Thus, we assigned the Gaussian component $S_{0}$ to solid matrix of the wheat thylakoid lyophilizate, and used this amplitude as a unit to scale the amplitudes of the other signal components.
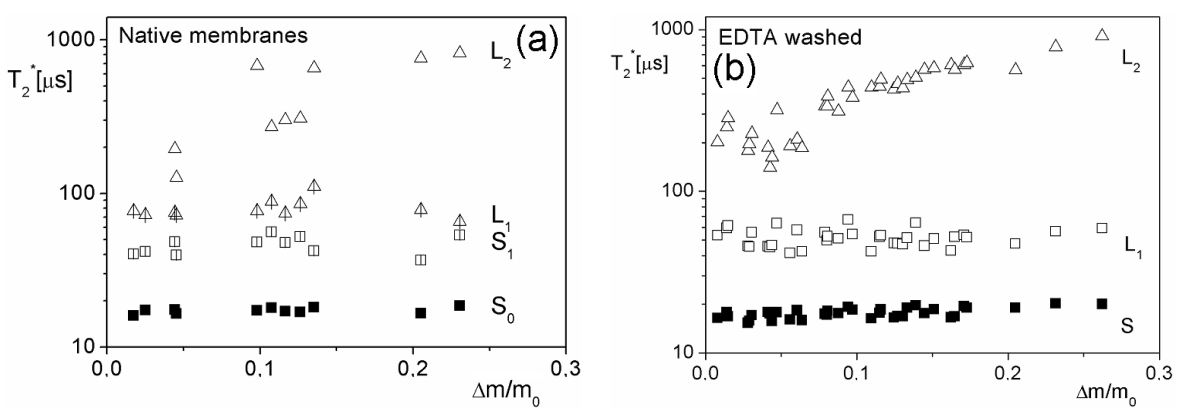

Fig. 3. Proton spin-spin relaxation times from FID for (a) native wheat photosynthetic membranes, and for (b) EDTA-washed membranes, and rehydrated from lyophilizate as a function of hydration level, $\Delta m / m_{0}$, in units of dry weight. Solid squares - Gaussian component $S_{0}$, dashed squares - Gaussian $S_{1}$, dashed triangles - exponential $L_{1}$, and open triangles - exponential $L_{2}$.

The amplitude of the second (ii) Gaussian component, $S_{1}$, linearly depends on the hydration level, according to the equation: $S_{1} / S_{0}=k \Delta m / m_{0}+S_{1.0} / S_{0}$ for native membranes, suggesting that this component comes from water bound to the surfaces of the lyophilizate (Fig. 4). The Gaussian form of the decay and the short value of $1 / \mathrm{e}$-time, $T_{S, 1}^{*}$, suggests that $S_{1}$ component comes from deeply immobilized fraction of water. As the value of $T_{S, 1}^{*}$ does not change within the whole hydration range investigated, and equals $\approx 50 \mu \mathrm{s}$, we suggest that it is a homogeneous fraction of water. The $S_{1}$ fraction is not detected in $1 \mathrm{mM}$ EDTA-washed membranes.

The exponentially decaying fraction $L_{1}$ (iii) also increases with the increasing hydration level and is well described by the linear dependence: $L_{1} / S_{0}=$ $k \Delta m / m_{0}+L_{1,0} / S_{0}$ either for native or EDTA-washed membranes (Fig. 4). Similarly to the fraction $S_{1}$, the value of the decay time does not depend much on 

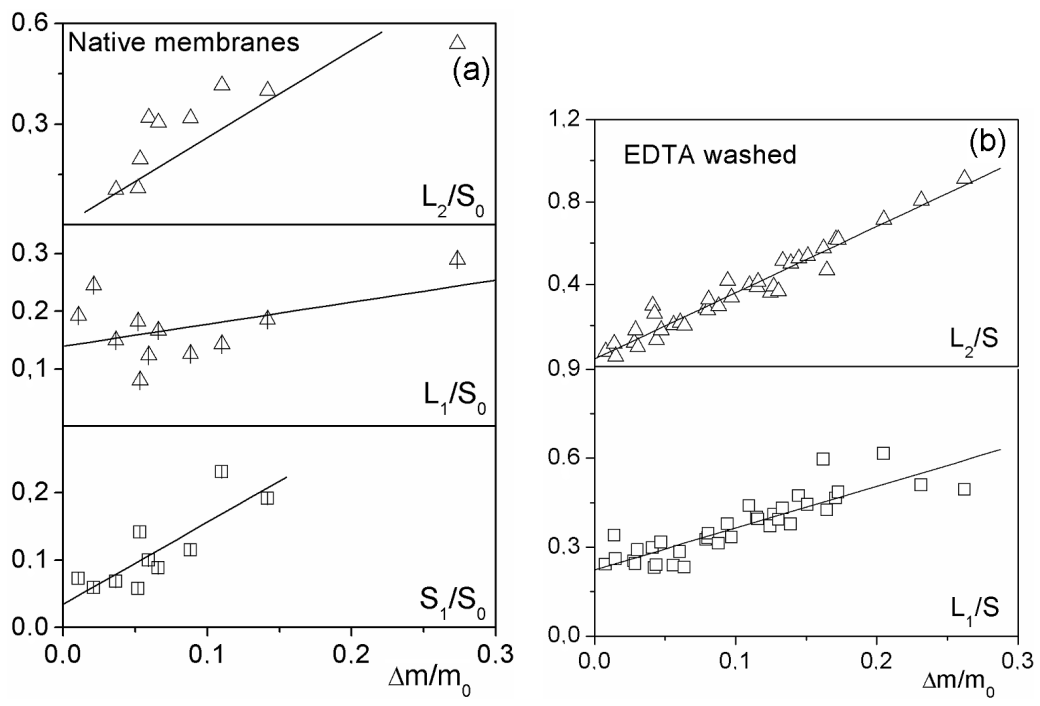

Fig. 4. The amplitudes of FID liquid components expressed in units of solid, $S_{0}$, recorded for (a) native, and (b) EDTA-washed wheat thylakoid membranes rehydrated from lyophilizate as a function of hydration level, $\Delta m / m_{0}$, in units of dry weight. Dashed squares - Gaussian $S_{1}$, dashed triangles — exponential $L_{1}$, and open triangles - exponential $L_{2}$.

the hydration level and equals $T_{L, 1}^{*} \approx 80 \mu \mathrm{s}$. Proton FID time for the $L_{1}$ fraction resembles that for the tightly bound water signal of lichen thalli $[21,4,22]$, dentine and dental enamel [24], bark and bast [23], and of the control porous glass [25]. These suggest that $L_{1}$ comes from water fraction tightly bound to the lyophilizate. High values of the errors of the slopes of the liquid signal hydration dependencies show that the amplitude of this component may saturate with increasing hydration level, however, the intercepts are estimated with sufficient accuracy.

The amplitude of the (iv) long exponential component, $L_{2}$, linearly increases with the increasing hydration level with the dependence $L_{2} / S_{0}=k \Delta m / m_{0}$ for the mature and for EDTA-washed membranes (Fig. 4). The proton FID time for the $L_{2}$ component increases with the increasing hydration level (Fig. 3), which means that this component is an average of loosely bound water fractions being in fast exchange regime [26]. The values of decay time are much longer than the ones for the components (i), (ii) and (iii), and are of the order of $T_{L, 2}^{*} \approx 1 \mathrm{~ms}$.

The recorded three water fractions are differentiated by their dynamics, thus by their proximity to the solid surface. However, the $T_{L, 2}^{*}$ value for loosely bound water is strongly shortened by $B_{0}$ inhomogeneities, preventing (by the specificity of FID measurement) the detailed analysis of the loosely bound water pools.

The non-zero contributions of both tightly bound water fractions, $S_{1,0}$ and $L_{1,0}$, show the presence of "sealed" water pool, which does not leave the wheat photosynthetic membrane during lyophilization procedure. Recalculated in units 
of dry mass, the contribution of sealed water equals $\Delta M_{s} / m_{0}=0.039 \pm 0.011$ and 0.048 for native and EDTA-washed membranes, respectively.

The hydration dependence of total liquid signal, $\left(S_{1}+L_{1}+L_{2}\right) / S_{0}$, in units of solid, is linear (see Fig. 5), suggesting the absence of water soluble solid fraction, because otherwise the hydration dependence would be described by the rational function $[27,23]$. The contribution of sealed water calculated from total liquid signal hydration dependence equals $\Delta M_{s} / m_{0}=0.045 \pm 0.009$ and $0.057 \pm 0.007$ for native and for EDTA-washed membranes, respectively.
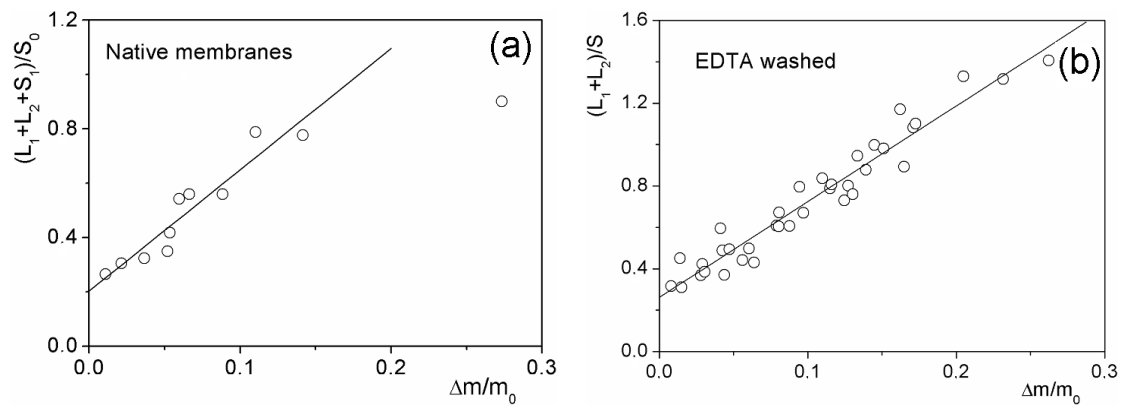

Fig. 5. The total liquid signal expressed in units of solid, $\left(S_{1}+L_{1}+L_{2}\right) / S_{0}$, for (a) native wheat thylakoid membranes, and (b) EDTA-washed membranes rehydrated from lyophilizate as a function of hydration level, $\Delta m / m_{0}$, in units of dry weight. The data for the developing membranes recorded for the highest hydration level was not taken to fit the straight line, because here the error of the $S_{0}$ value is the highest and may influence the resulting value of total liquid signal.
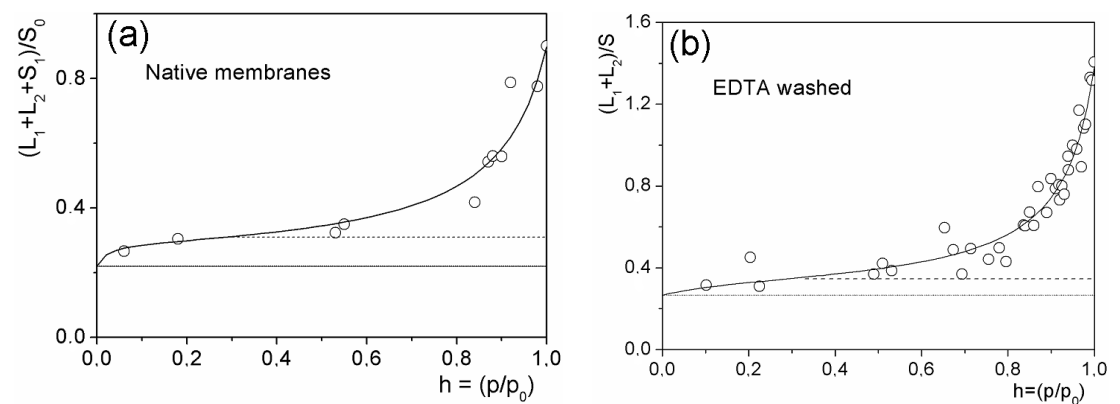

Fig. 6. The total liquid signal in units of solid, $\left(S_{1}+L_{1}+L_{2}\right) / S_{0}$ versus humidity $h$ for (a) native wheat thylakoid membranes and (b) EDTA-washed membranes, rehydrated from lyophilizate. Solid line - the sorption isotherm from gravimetric data, linearly fitted to the NMR data. Fitted parameters: $\left(S_{1,0}+L_{1,0}\right) / S_{0}-$ the contribution of "sealed" water, and $k$ - proportionality coefficient between amplitude of the liquid NMR signal and the mass of the water added. Dotted line shows the level of the "sealed" water, $\left(S_{1,0}+L_{1,0}\right) / S_{0}$, whereas dashed line - the level of primary binding site population. 
The total proton liquid signal, $\left(S_{1}+L_{1}+L_{2}\right) / S_{0}$, was used to construct the NMR-isotherm (Fig. 6), where the values $\Delta M / m_{0}, b$, and $b_{1}$ were not fitted but taken from fits of sorption isotherm

$$
\frac{S_{1}+L_{1}+L_{2}}{S_{0}}(h)=\frac{S_{1,0}+L_{1,0}}{S_{0}}+k \frac{\Delta M}{m_{0}} \frac{b_{1} h}{(1-b h)\left(1+b_{1} h-b h\right)} .
$$

So obtained sorption isotherm fits the NMR data sufficiently well and the value averaged over all methods $\left(S_{1,0}+L_{1,0}\right) / S_{0}=0.199$, and $0.251\left(\Delta M_{s} / m_{0}=\right.$ $0.052 \pm 0.019$ and $0.061 \pm 0.006$ ), whereas $k=3.73$ and 4.63 for native and for EDTA-washed membranes, respectively.

\section{Discussion}

The mass of water saturating the primary water binding sites, $\Delta M / m_{0}$, in the preparation of the mature lyophilized photosynthetic membranes is significantly lower than in dry lichen thalli [17], and still decreased by washing out the loosely bound paramagnetic ions ( $1 / b_{1}$ increases from $2.2 \%$ to $\left.14.6 \%\right)$. This suggests that the surface of such dry photosynthetic membranes is more hydrophobic than the membrane systems of lichen thallus, and bound paramagnetic ions increase the number of bound water molecules.

The ordering (decreased partial molar volume and entropy) of water molecules surrounding a hydrated paramagnetic ion was already suggested for ions in solution $[28,29]$. If an ion is bound on the surface, the water immobilization may be so high that the FID component relaxes in the form of Gaussian function.

Proton NMR data revealed the presence of significant fraction of water, which is retained in dry lyophilizate. Its mass in units of $m_{0}$ and averaged over the values obtained using several approaches, equals $\Delta M_{s} / m_{0}=0.052 \pm 0.019$ for native membranes, and $\Delta M_{s} / m_{0}=0.061 \pm 0.006$ for EDTA-washed membranes. For both types membranes the mass of sealed water exceeds twice the contribution of water saturating primary water binding sites, $\Delta M / m_{0}$.

Precise localization of these "sealed" water pools needs further research. Probably this could be water occluded in lumenal space of thylakoid vesicles. Another possibility are supramolecular structures formed from proteins and lipids. A good candidate for water-encapsulating lipid structures could be the tubules of inverted hexagonal phase formed by monogalactosyldiacylglycerol (MGDG), a dominating lipid species of the photosynthetic membranes. Domains enriched in inverted hexagonal phases have been detected both in the native photosynthetic membranes [30] as well as in model phosphatidylcholine liposomes containing MGDG [31]. However, the question if such structures present in fully hydrated membranes also occur in lyophilized thylakoids, remains to be found. 


\section{References}

[1] H.J. Bohnert, E. Sheveleva, Curr. Opin. Plant Biol. 1, 267 (1998).

[2] F. Navarizzo, F. Ricci, C. Vazzana, M.F. Quartacci, Physiol. Plant. 94, 135 (1995).

[3] K. Schneider, B. Wells, E. Schmelzer, F. Salamini, D. Bartels, Planta 189, 120 (1993).

[4] H. Harańczyk, S. Gaździński, M. Olech, New Aspects in Cryptogamic Research, Contribution in Honour of Ludger Kappen. Bibl. Lichenol. 75, 265 (2000).

[5] H. Harańczyk, J. Grandjean, M. Olech, Coll. Surf. B, Biointerfaces 28, 239 (2003).

[6] H. Harańczyk, J. Grandjean, M. Olech, M. Michalik, Coll. Surf. B, Biointerfaces 28, 251 (2003).

[7] L. Kappen, Arctic 46, 297 (1993).

[8] B. Schroeter, Ch. Scheidegger, New Phytol. 131, 273 (1995).

[9] H. Harańczyk, K. Strzałka, T. Bayerl, G. Klose, J.S. Blicharski, Photosynthetica 19, 414 (1985).

[10] H. Harańczyk, K. Strzałka, W. Dietrich, J.S. Blicharski, J. Biol. Phys. 21, 125 (1995).

[11] H.H. Robinson, R.R. Sharp, C.F. Yocum, Biochem. Biophys. Res. Commun. 93, 755 (1980).

[12] T.J. Wydrzynski, S.B. Marks, P.G. Schmidt, Govindjee, H.S. Gutowsky, Biochemistry 17, 2155 (1978).

[13] T.J. Wydrzynski, N. Zumbulyadis, P.G. Schmidt, Govindjee, Biochim. Biophys. Acta 408, 349 (1975).

[14] K. Strzałka, G. Majewska, E. Mędrela, Acta Physiol. Plant. 11, 49 (1980).

[15] D.F. Gaff, Oecologia (Berl.) 31, 95 (1977).

[16] W. Wȩglarz, H. Harańczyk, J. Phys. D, Appl. Phys. 33, 1909 (2000).

[17] H. Harańczyk, On Water in Extremely Dry Biological Systems, Wydawnictwo Uniwersytetu Jagiellońskiego, Kraków 2003.

[18] A.J. Hailwood, S. Horrobin, Trans. Faraday Soc. B 42, 84 (1946).

[19] R.W. Dent, Textile Res. J. 47, 145 (1977).

[20] S. Brunauer, P.H. Emmett, E. Teller, J. Am. Chem. Soc. 60, 309 (1938).

[21] H. Harańczyk, S. Gaździński, M.A. Olech, New Phytologist 138, 191 (1998).

[22] H. Harańczyk, S. Gaździński, M. Olech, Mol. Phys. Reps. 29, 135 (2000).

[23] H. Harańczyk, W.P. Wȩglarz, S. Sojka, Holzforschung 53, 299 (1999).

[24] N. Funduk, G. Lahajnar, L. Miljković, S. Skocajić, D.W. Kydon, L.J. Schreiner, M.M. Pintar, Zobozdrav. Vestn. 41 (Suppl.1), 139 (1986).

[25] H. Harańczyk, K.G. Soga, R.J. Rumm, M.M. Pintar, Magn. Res. Imag. 9, 723 (1991).

[26] J.R. Zimmerman, W.E. Brittin, J. Phys. Chem. 61, 1328 (1957).

[27] H. Harańczyk, K. Strzałka, G. Jasiński, K. Mosna-Bojarska, Coll. Surf. A 115, 47 (1996) 
[28] H.S. Frank, M.W. Evans, J. Chem. Phys. 13, 507 (1945).

[29] C. Tanford, The Hydrophobic Effect: Formation of Micelles and Biological Membranes, Wiley, New York 1986, p. 27.

[30] K. Gounaris, A. Sen, A.P.R. Brain, P.J. Quinn, W.P. Williams, Biochim. Biophys. Acta 728, 129 (1983).

[31] D. Latowski, J. Kruk, K. Burda, M. Skrzynecka-Jaskier, A. Kostecka-Gugała, K. Strzałka, Eur. J. Biochem. 269, 4656 (2002). 